\title{
Pulse Artifact Detection in Simultaneous EEG-fMRI Recording Based on EEG Map Topography
}

\author{
Giannina R. Iannotti • Francesca Pittau • \\ Christoph M. Michel · Serge Vulliemoz • \\ Frédéric Grouiller
}

Received: 26 May 2014/Accepted: 8 October 2014/Published online: 12 October 2014

(C) Springer Science+Business Media New York 2014

\begin{abstract}
One of the major artifact corrupting electroencephalogram (EEG) acquired during functional magnetic resonance imaging (fMRI) is the pulse artifact (PA). It is mainly due to the motion of the head and attached electrodes and wires in the magnetic field occurring after each heartbeat. In this study we propose a novel method to improve PA detection by considering the strong gradient and inversed polarity between left and right EEG electrodes. We acquired high-density EEG-fMRI (256 electrodes) with simultaneous electrocardiogram (ECG) at $3 \mathrm{~T}$. PA was estimated as the voltage difference between right and left signals from the electrodes showing the strongest artifact (facial and temporal). Peaks were detected on this estimated signal and compared to the peaks in the ECG recording. We analyzed data from eleven healthy subjects, two epileptic patients and four healthy subjects with an insulating layer between electrodes and scalp. The accuracy of the two methods was assessed with three criteria: (i) standard deviation, (ii) kurtosis and (iii) confinement into the physiological range of the inter-peak intervals. We also checked whether the new method has an influence on the identification of epileptic spikes. Results show that
\end{abstract}

G. R. Iannotti - F. Pittau - C. M. Michel - S. Vulliemoz Functional Brain Mapping Laboratory, Department of Fundamental Neurosciences, Geneva University Hospital, 1211 Geneva 14, Switzerland

F. Pittau $\cdot$ S. Vulliemoz

EEG and Epilepsy Unit, Department of Neurology, Geneva

University Hospital, 1211 Geneva 14, Switzerland

F. Grouiller $(\square)$

Department of Radiology and Medical Informatics, Geneva

University Hospital, Rue Gabrielle-Perret-Gentil 4,

1211 Geneva 14, Switzerland

e-mail: frederic.grouiller@hcuge.ch estimated PA improved artifact detection in 15/17 cases, when compared to the ECG method. Moreover, epileptic spike identification was not altered by the correction. The proposed method improves the detection of pulse-related artifacts, particularly crucial when the ECG is of poor quality or cannot be recorded. It will contribute to enhance the quality of the EEG increasing the reliability of EEGinformed fMRI analysis.

Keywords EEG-fMRI · High-density EEG · Gradient artifact - Ballistocardiogram - Pulse artifact detection . EEG topography

\section{Introduction}

The combination of Electroencephalography (EEG) and functional magnetic resonance imaging (fMRI) has become an important tool for non-invasive investigation of human brain functions, offering at the same time the high temporal resolution of the EEG and the high spatial resolution of fMRI. The use of this technique is increasingly spreading across cognitive neuroscience (Debener et al. 2006; Zotev et al. 2014), sleep studies (Czisch et al. 2004; Stern et al. 2011), and resting state networks investigation (Britz et al. 2010; Neuner et al. 2014). Concerning epilepsy, EEGfMRI allows the characterization of focal and generalized epileptiform discharges and is typically applied for the presurgical localization of epileptic foci (Pittau et al. 2014).

The EEG signal recorded inside the scanner is highly artifacted and several steps of EEG pre-processing are needed to allow the detection of cerebral electrical activity. Two major kinds of MR-related artifacts affect the EEG: (i) gradient (or imaging) artifact (GA), due to the time-varying magnetic field gradients (Allen et al. 2000; 
Felblinger et al. 1999; Yan et al. 2009); (ii) pulse artifact (PA), that mainly derives from the pulsation of the blood in scalp arteries causing electrodes movement inside the static magnetic field of the scanner. GA has huge amplitude (of the order of $\mathrm{mV}$, almost 1,000 times higher than cerebral signal), but given its strict periodicity and stability, it can be corrected reliably, using moving average artifact waveform subtraction (Allen et al. 2000). In contrast to the GA, the PA is more difficult to remove because of its characteristics: (i) it is highly non stationary (its duration and amplitude vary between successive heartbeats), (ii) most of its power lies in EEG frequency range $(1-50 \mathrm{~Hz})$; (iii) it depends on the spatial configuration and orientation of electrodes in the magnetic field; (iv) it varies across subjects; (v) it is proportional to the scanner static magnetic field strength (Debener et al. 2008).

The physical mechanisms generating the PA have been studied (Mullinger et al. 2013; Yan et al. 2010). PA is a consequence of the motion of the electrodes and wires in the magnetic field following each heartbeat. It induces an electromotive force that depends on the orientation of the electrodes related to the magnetic field and adds to the voltage potential measured by electrodes. The strongest contribution to electrode motion comes from the nodding head motion (pitch) caused by the quick arrival of blood in the head arteries as demonstrated by a strong reduction of the PA by the use of a bite-bar (Mullinger et al. 2013). Blood from scalp arteries likely contributes more than blood from the large intracranial arteries (Yan et al. 2010) as suggested by discrepancies between simulation and experimental data. Another contribution, smaller than the previous one, to the PA is due to the conductive nature of pulsatile blood in the head interacting with the magnetic field (Hall Effect). The contribution of pulsatile scalp expansion to the electrode motion is also present but it has not been as thoroughly investigated as both former contributions. In the usual practice the nodding and the pulse driven scalp expansion effects can be reduced using cushions to restrict the head. In contrast, the use of an insulating layer that constitutes the only demonstrated way to exclude the Hall Effect contribution (Mullinger et al. 2013), is obviously not adoptable since it will mask the brain activity.

The PA occurs approximately $210 \mathrm{~ms}$ after the QRS complex because of the displacement of the blood from the heart to the head. The standardized strategy to correct PA relies on Average Artifact Subtraction, AAS (Allen et al. 1998), which can be complemented by estimating an Optimal Basis Set of principal components (Niazy et al. 2005) and/or by an ICA (Independent Component Analysis) decomposition to remove residual artifacts (Debener et al. 2007; Vanderperren et al. 2010). Other authors proposed a direct ICA on EEG (Benar et al. 2003; Marques et al. 2009). However, PA is not spatially stationary which contravenes with ICA assumptions and could explain incomplete removing of PA with ICA (Debener et al. 2008). Spatial filtering of the EEG using beamformer has also been proposed in addition to AAS, to remove residuals of GA and PA (Brookes et al. 2008).

The methods based on average artifacts subtraction (i.e. AAS, OBS) relies on the precise detection of the QRS complex of the ECG signal and make the assumption that the delay between ECG and PA is constant. Indeed, the presence of a jitter or wrong detection could critically reduce the performance of the artifacts correction based on moving average template subtraction (Grouiller et al. 2007). In addition to the intra-subject beat-to-beat variability of the QRS complexes, another difficulty in detecting the ECG relies on various types of noise that can corrupt the ECG signal (muscle activity, cardiac electrodes motion related to respiration, helium pump artifacts, gradient artifacts residuals, incidental detachments of the electrodes from the chest, large breasts). Furthermore, the T-wave following the QRS complex can be amplified and distorted as the magnetic field increases (Debener et al. 2008). This may be due to the simultaneous occurrence of the T-wave with the opening of the aortic valve and subsequent ejection of blood into the aorta (Weikl et al. 1989). All these effects result in a less sharp and degraded ECG signal, impeding proper AAS.

Considering the scalp voltage map of the pulse artifact, it has been demonstrated that it varies in topography and magnitude across time (Yan et al. 2010) and evolves as a succession of typical voltage maps characterized by reversed polarities over left and right hemisphere. The amplitude of the PA is generally higher in the temporal electrodes and the polarity is negative on the left and positive on the right (Yan et al. 2010). The aim of the present study is to directly detect PA on the scalp using this characteristic topography. Thereby we take advantage of a good spatial sampling of the basal temporal and facial area. Some previous groups suggested to cross-correlate a PA artifact template (Mijovic et al. 2012) or a signal from a sensor (Shin et al. 2008) to detect markers. We propose a novel and simple method to detect peaks of PA for subsequent AAS correction in order to improve the quality of EEG data acquired during fMRI. Our method does not require ECG or any additional hardware.

\section{Materials and Methods}

We developed an EEG-based method for pulse artifact detection taking advantage of its clear scalp voltage topography. We performed several evaluation steps: (i) comparison of the new method with the standard ECG- 
based detection in eleven healthy subjects, in two epileptic patients and in four subjects with electrically insulating layer between scalp and electrodes; (ii) evaluation of the benefit of recordings with different number of electrodes, i.e. large number of temporal and facial electrodes that covers areas presenting highest voltage asymmetry versus less electrodes; (iii) assessment of possible alteration of the EEG after PA correction using the new method. To perform the last point, GA and PA artifacts from a subject with insulating layer between scalp and EEG electrodes were added to a high-density EEG from an epileptic patient acquired outside the scanner which was then corrected using our technique. Two electroencephalographers, blind to each other, then identified spikes on the native and artifacted/corrected recordings.

\section{Subjects}

We recruited: eleven healthy volunteers with no history of neurological or cardiological disorders (H1-H11, 5 females, mean age $=31$-year-old), two patients with refractory epilepsy (P1-P2, 1 male, age $=12$ - and 56-yearold), recruited in the Neurology Department of Geneva University Hospital (Switzerland) and four healthy subjects with insulating layer between scalp and EEG electrodes (A1-A4). Written informed consent was obtained prior to the experiment, in accordance with the local ethics committee guidelines.

In the subjects with insulating layer between scalp and electrodes, we recorded the pulse artifact related to electrode motion in the magnetic field without any other EEG activity similarly to previous studies (Mullinger et al. 2013). For this purpose, we electrically insulated the scalp with a swimming cap extended on the cheeks by plastic gloves. As a conductive layer we placed a cloth saturated with saline solution between the swimming cap and the EEG net. The use of this insulating layer allows to remove brain signal and Hall effect.

\section{Data Acquisition}

\section{High-Density EEG and ECG}

We recorded electrical activity using an MR-compatible EEG system (Electrical Geodesic, Inc., Eugene, Oregon) made up of 256 electrodes that includes a large number of electrodes on lower temporal areas and cheeks. Each electrode contains a small sponge soaked with saline solution that make contact with the patient's scalp. The skin of the subjects was scrubbed and an elastic bandage was placed above the EEG cap to maintain the contact of electrodes on the scalp. For safety reason, each electrode is equipped with an additional $10 \mathrm{k} \Omega$ resistance (Lemieux et al. 1997). The electrodes impedance was kept as low as possible (usually $<40 \mathrm{k} \Omega$, median \pm standard deviation: $22.1 \pm 10.1 \mathrm{k} \Omega$ ). During simultaneous EEG-fMRI recordings we also acquired the electrocardiogram (ECG) signal with two MR-compatible electrodes positioned on the chest, each on one side of the heart. The EEG and ECG cables passed through the front end of the bore (patient's feet) to the MR-compatible amplifier positioned to the left side of the participant. The EEG was synchronized to the MRI clock and acquired at $1 \mathrm{kHz}$ sampling frequency (Mandelkow et al. 2006).

\section{$f M R I$}

EEG-fMRI recordings were performed in a $3 \mathrm{~T}$ scanner (Siemens Magnetom Trio, 12-channel head receive coil with body transmit coil) using a $\mathrm{T}_{2}^{*}$-weighted single-shot gradient echo echo-planar images (repetition time $[\mathrm{TR}]=1990 \mathrm{~ms}$, $6 \mathrm{~ms}$ of delay, echo time $[\mathrm{TE}]=30 \mathrm{~ms}, 32$ slices, $64 \times 64$ matrix, voxel size $3 \times 3 \times 3.75 \mathrm{~mm}^{3}$, flip angle $=90$ degrees, continuous and descending acquisition).

During the recording, all lights inside the scanner room and the internal ventilation system of the MR scanner were turned off to reduce the contamination of the EEG by environmental artifacts (Nierhaus et al. 2013). We immobilized the head of volunteers with a memory foam pillow in order to reduce the effect of nodding in the PA.

For healthy subjects and subjects with insulating layer between scalp and electrodes, we acquired 150 volumes ( 5 min of recording) whilst for each epileptic patient we extracted $5 \mathrm{~min}$ out of a resting state recording of $20 \mathrm{~min}$.

\section{Data Analysis}

All analyses were performed using an in-house code written in Matlab (The MathWorks, Inc., MA, USA) and results were visualized with CARTOOL software (http:// sites.google.com/site/fbmlab/cartool) (Brunet et al. 2011).

\section{Gradient Artifact Correction}

Gradient artifact removal was performed using a moving averaged template subtraction method, followed by artifact residual removal using optimal basis set and adaptive noise cancellation (Allen et al. 1998; Niazy et al. 2005).

\section{EEG-Based and ECG-Based Pulse Artifact Detection}

Figure 1 shows the scalp voltage topography at the peak of PA, estimated from the EEG averaged with respect to the ECG markers after gradient artifact correction. We selected the electrodes with the strongest PA amplitude and highest inter-hemispheric voltage gradient due to their 
Fig. 1 Topographic voltage map of the averaged pulse artifact (PA). Electrodes showing the strongest PA amplitude are considered for the estimation of the BCG signal and are highlighted in pink (Color figure online)

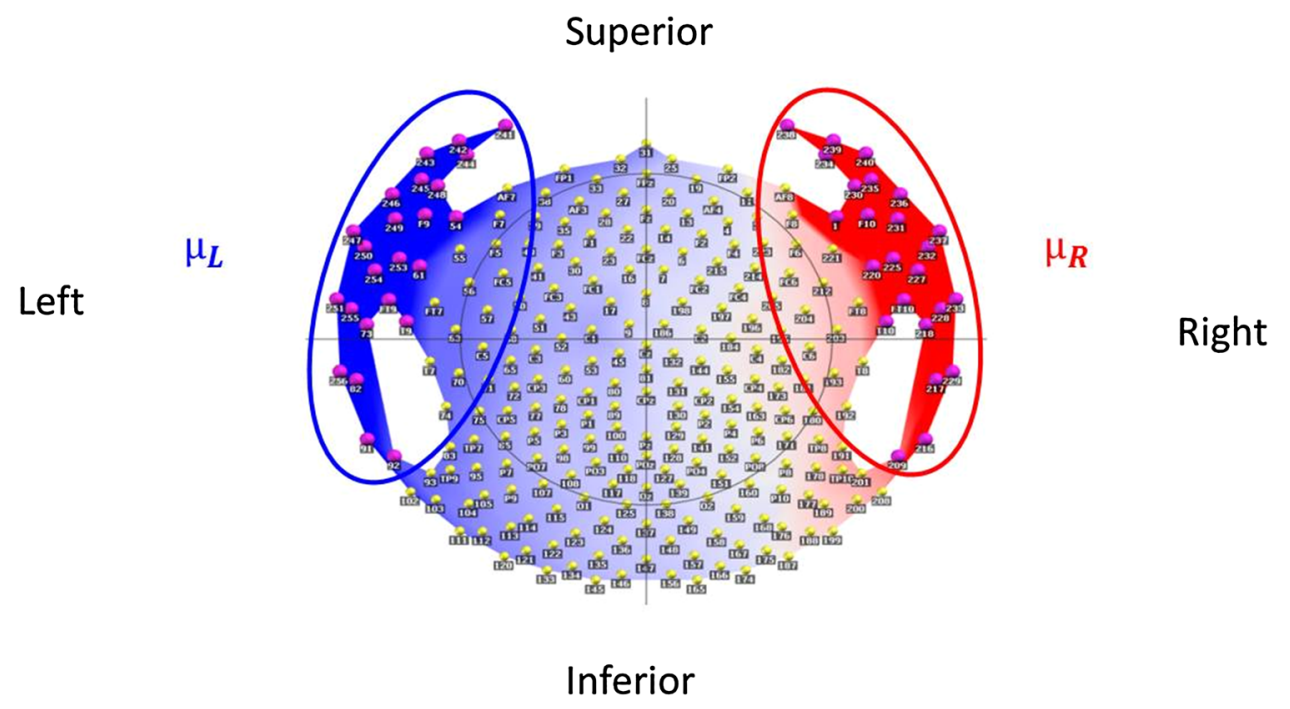

orientation into the magnetic field (24 channels per side, marked in pink in Fig. 1). These electrodes are located on the right and left anterior temporal and facial regions. Taking advantage of this strong polarity difference, we estimated the PA as the difference between the averaged EEG signals of electrodes on the right $\left(\mu_{R}\right)$ and on the left $\left(\mu_{L}\right)$. The resulting trace is an estimation of the ballistocardiogram and is referred to as the BCG signal:

$B C G=\mu_{R}-\mu_{L}$

The detection of the peak was performed on both the ECG and the BCG following a method elaborated to identify the heart QRS complex on an ECG signal (Christov 2004). The detection algorithm was applied to the gradient-corrected signal. We evaluated the time between consecutive markers for ECG and BCG, $\Delta_{\mathrm{ECG}}$ and $\Delta_{\mathrm{BCG}}$. We then used three criteria to assess the reliability of BCG and ECG-based detection of the pulse artifact:

- The standard deviation $\sigma$ of $\Delta_{\mathrm{ECG}}$ and $\Delta_{\mathrm{BCG}}$ divided by their respective mean $\mu, \sigma / \mu$. We estimated that when the value of $\sigma / \mu$ is small, the peak detection is more regular and thus more reliable.

- The Kurtosis (K) of $\Delta_{\mathrm{ECG}}$ and $\Delta_{\mathrm{BCG}}$ to estimate the degree of sharpness of their distribution. In the current case, if the marker detection is good, we expect a narrow distribution and a high value of kurtosis. This criterion is a way to quantify the proportion of false positive or false negative that alters the sharpness of the distribution.

- The number L of $\Delta_{\mathrm{ECG}}$ and $\Delta_{\mathrm{BCG}}$ values, that are out of the physiological range (between 600 and $1,200 \mathrm{~ms}$ corresponding to a heart rate of 50-100 heartbeats per minute). The signal with lower $\mathrm{L}$ is considered to be of better quality.
Two out of the three conditions mentioned above had to be satisfied to define the best signal for the detection of pulse artifact peaks. Even when the ECG was chosen as the best signal, the BCG signal was useful for estimating a subject-specific constant delay between ECG and BCG, necessary for the subsequent EEG cardiac correction based on the average artifact subtraction (AAS) method.

We evaluated the quality of peak detection by checking manually the marker positions and calculating the percentage of missing (false negative, $\mathrm{F}_{\mathrm{N}}$ ) and wrong (false positive, $\mathrm{F}_{\mathrm{P}}$ ) markers for both signals. Any marker detected in the absence of a peak was considered as false positive, $\mathrm{F}_{\mathrm{P}}$. If the marker is shifted in time respect to the peak, we considered it both as $\mathrm{F}_{\mathrm{N}}$ and as $\mathrm{F}_{\mathrm{P}}$. If the marker is correctly positioned on the peak it is considered as a $T_{\mathrm{P}}$. We calculated the sensitivity, $S_{e}$, and the specificity, $S_{p}$, for each participant and for both signals as reported in (Niazy et al. 2005):

$S_{e}=\frac{T_{p}}{T_{p}+F_{N}}$

$S_{p}=\frac{T_{p}}{T_{p}+F_{p}}$

We use this sensitivity as a qualitative measure of the ECG. We considered the ECG of "High quality" if the sensitivity of ECG in detecting QRS peaks is higher than $90 \%$, "Intermediate quality" if the sensitivity is between 70 and $90 \%$ and "Low quality" if sensitivity is below $70 \%$.

In order to characterize the evolution in time of voltage topographic maps, two global measures are commonly used: the global field power (GFP) and the global map dissimilarity (GMD) (Lehmann and Skrandies 1980). 
The GFP is a measure of field strength, computed as the standard deviation of all electrodes referred to the average reference $($ mean $=0)$ :

$G F P=\sqrt{\frac{\sum_{i}^{N}\left(u_{i}-\hat{u}\right)^{2}}{N}}$

where $u_{\mathrm{t}}$ is the voltage potential at the electrode $i, \hat{u}$ is the average voltage potential of all electrodes and $N$ is the total number of electrodes.

A topographic map with a pronounced gradient will result in high GFP, while GFP is low in maps which have a "flat" appearance. Displaying of GFP measure over time allows the identification of moments of high signal-tonoise ratio (Koenig and Melie-Garcia 2010; Skrandies 2007).

The Global Map Dissimilarity is a measure of the difference between two topographic maps and it is defined as:

$G M D=\sqrt{\frac{1}{N} \sum_{1}^{N}\left\{\frac{u_{i}-\hat{u}}{\sqrt{\sum_{i}^{N} \frac{\left(u_{i}-\hat{u}\right)^{2}}{N}}}-\frac{v_{i}-\hat{v}}{\sqrt{\sum_{i}^{N} \frac{\left(v_{i}-\hat{v}\right)^{2}}{N}}}\right\}^{2}}$

where $u$ and $v$ indicate values for the two maps.

The GMD is equivalent to the spatial Pearson's productmoment correlation coefficient between the potentials of the two maps to compare (Brandeis et al. 1992). The GMD can be used to compare topographies between successive time points. The display of the GMD across time then allows defining periods of map stability and moments of map changes. It is generally observed that GMD is inversely correlated with the GFP: GMD is high when GFP is low (Lehmann et al. 1987). Commonly, the GFP peak is confined between two peaks of the GMD, indicating that maps tend to be stable at periods of high GFP and change configuration at time points of low GFP.

In our analysis, after manual correction of wrong and missing markers, we used these two parameters to characterize the topographic maps obtained by averaging the gradient corrected EEG with respect to all of the ECG and all of the PA markers, respectively. For a matter of simplicity, we referred to them as $E E G_{E C G}$ and $E E G_{P A}$ respectively.

For each subject, we determined the GFP peak of the averaged signal in a time window of $\pm 250 \mathrm{~ms}$ around the marker. The higher the amplitude of the GFP peak, the more accurate the detection of the peak used for the averaging. We also compared the widths of the GFP on the two averaged signals, estimating the difference between the nearest peaks of the GMD around the GFP maximum peak. In fact, the more accurate the peak detection, the narrower the GFP peak and the smaller the time between peaks of GMD. GFP peaks amplitude and width were compared for ECG and BCG detection using a paired two-sided
Wilcoxon signed-rank test to assess the accuracy of the detection. In the $E E G_{E C G}$ map, the difference in time between the maximum value of GFP and the ECG onset ('Origin', see Fig. 2) represents the mean latency of the PA peaks related to the heartbeats.

Figure 2 shows as example the EEG of a subject with an insulating layer between scalp and electrodes (A1) corrected for gradient artifact and then averaged with respect to the ECG markers $\left(E E G_{E C G}\right)$. The maximum of the GFP is delayed in time with respect to the ECG onset ("Origin") whilst it coincides with the onset of the corrected EEG averaged with respect to the PA markers (the $E E G_{P A}$ signal). For a better comprehension, we reported the $E E G_{P A}$ onset ('PA_peak' in Fig. 2) on the $E E G_{E C G}$ signal. The time delay between the two onsets indicates that the pulse artifact is delayed compared to the ECG events. This latency is not perfectly constant within a subject and it varies across subjects as shown in Fig. 3.

\section{Comparison with Reduced Number of Electrodes}

Many centers use EEG-fMRI with 64-channels EEG. Thus, for the estimation of the BCG signal and for the PA peak detection, we compared the complete temporo-facial electrodes coverage (see Fig. 1) with a lower sampling subset of electrodes including only four electrodes that are present in the usual 64 channels system: FT9, TP9, FT10 and TP10 (Fig. 4).

\section{Effect of the Correction on Epileptic Activity}

We investigated the effect of EEG-based pulse artifact detection on the identification of temporal epileptic spikes by simulating a simultaneous EEG-fMRI dataset of a patient. For this purpose we selected an epileptic patient with right temporal subcortical heterotopia who presented independent right anterior temporal and right temporooccipital spikes on high-density EEG recording outside the MRI. The simulated intra-scanner EEG was obtained by adding the recording of the EEG in a subject with an insulating layer between scalp and electrodes (A1), $E E G_{\text {insulated }}$, to the EEG signal of the patient acquired outside the scanner, $E E G_{\text {out }}$ :

$E E G_{\text {simul_in }}=\left(E E G_{\text {out }}+E E G_{\text {insulated }}\right)$

As the $E E G_{\text {insulated }}$ adds gradient and cardiac artifacts to the EEG outside, their removal leads to an estimation of the original EEG acquired outside as represented in the following:

${\widehat{E E G_{\text {out }}}}=E E G_{\text {corrected }}=E E G_{\text {simul_in }}-$ Artifacts

Two expert neurologists (F.P and S.V.) were asked to separately mark spikes on the $E E G_{\text {out }}$ and on the 


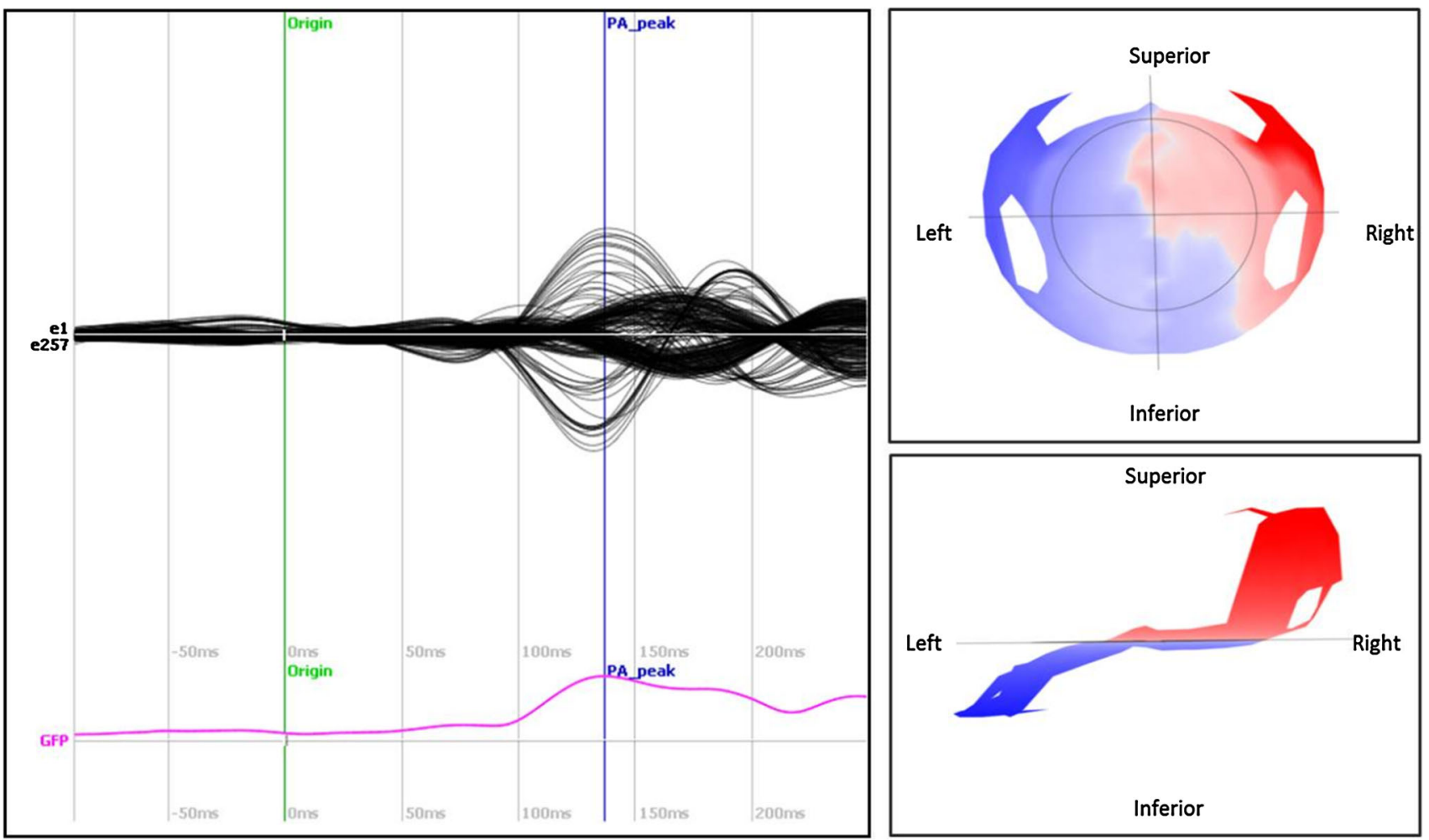

Fig. 2 Averaged PA in a subject with an insulating layer between scalp and electrodes. On the left, the gradient corrected EEG averaged respect to the ECG markers $\left(E E G_{E C G}\right)$. The 'Origin' represents the onset of the ECG events; the blue marker "PA_peak" reports on the same signal the onset of the corrected EEG averaged with respect to the PA peaks $\left(E E G_{P A}\right)$. On the right the $2 \mathrm{D}($ top $)$ and 3D (bottom) topographic map at the maximum of the GFP (PA_peak) (Color figure online)

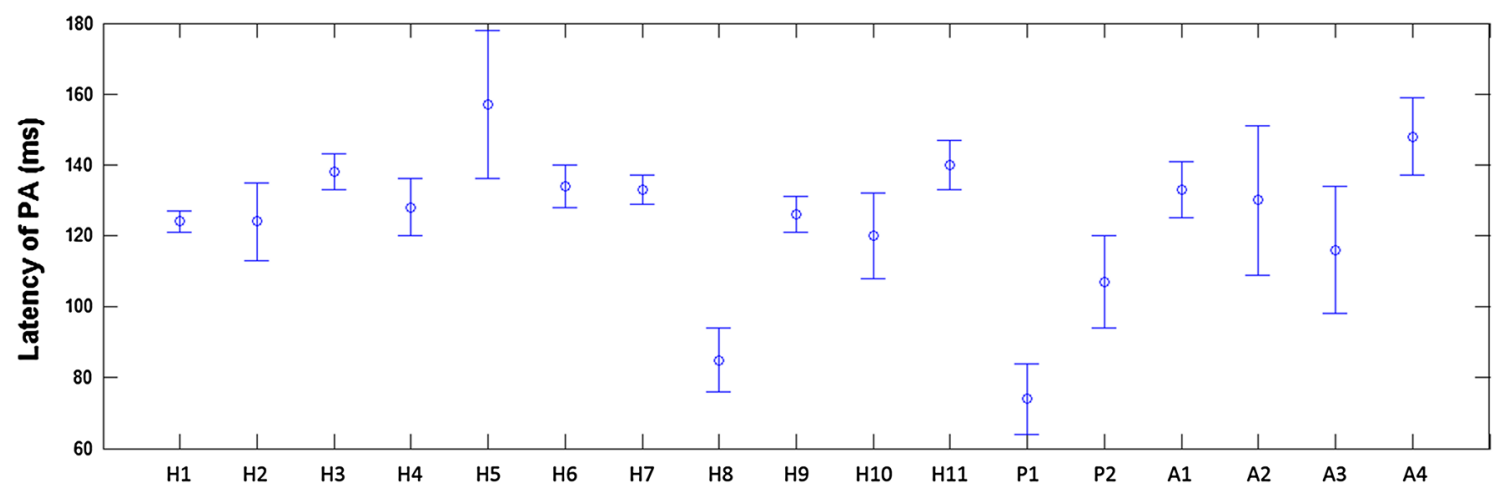

Fig. 3 Inter and intra-subject variability of the latency between ECG peaks and PA. The graph shows for each subject the mean value and the standard deviation of the time delay between the heartbeat and the pulse artifact on the scalp

$E E G_{\text {corrected }}$. We used the spikes commonly detected by the neurologists on both datasets to compute respectively the average of the $E E G_{\text {out }}$ and of the $E \widehat{E G}_{\text {out }}$ signals and obtain the related scalp voltage maps.

\section{Results}

The best signal for the peaks artifact detection was selected according to the criteria $\sigma / \mu, \mathrm{K}$ and $\mathrm{L}$, defined in the methods section. As shown in Table 1, the EEG-based approach outperformed the ECG-based one in 15 out of 17 cases with at least 2 out of 3 criteria favoring BCG over ECG.

Values for the sensitivity and specificity were respectively $97 \%( \pm 3 \%)$ and $98 \%( \pm 2 \%)$ for the BCG and $76 \%( \pm 27 \%)$ and $49 \%( \pm 26 \%)$ for ECG detection. The high standard deviations associated to the ECG sensitivity and specificity was due to the wide variability across subjects. By contrast, the BCG detection was very accurate. 


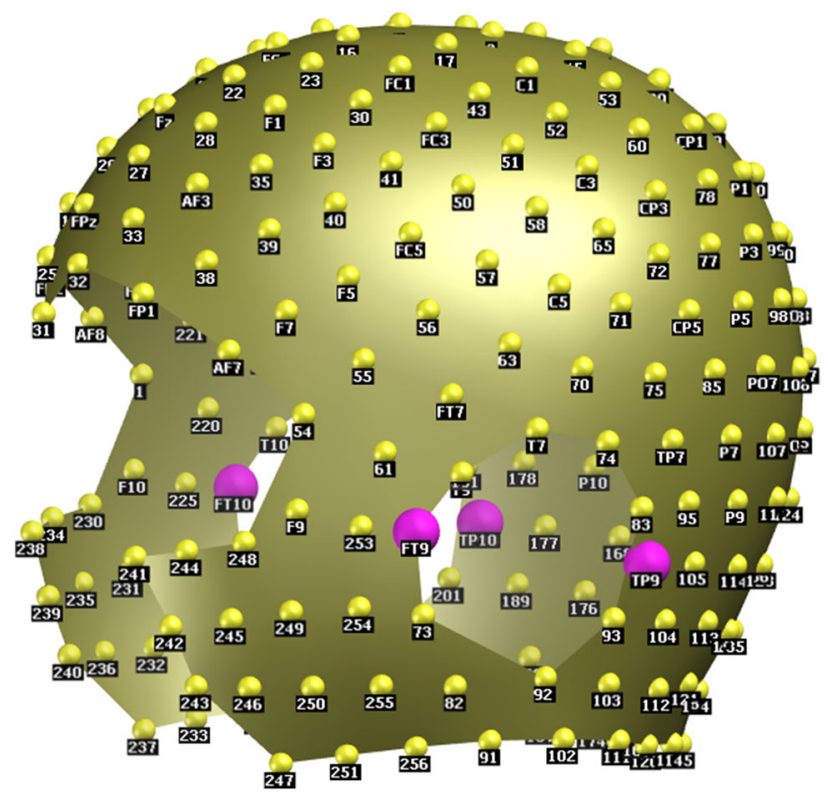

Fig. 4 Reduced subset of electrodes present in the usual 64 channels system used for the comparison between complete and reduced sets of electrodes. Electrodes highlighted in pink (FT9, TP9, FT10 and TP10) are used in the reduced set (Color figure online)

Table 1 Evaluation of the best detection with respect to each of the three criteria (standard deviation $\sigma / \mu$, kurtosis $\mathrm{K}$ and confinement into the physiological range $\mathrm{L}$ of the inter-peaks intervals)

\begin{tabular}{llll}
\hline Subjects & $\sigma / \mu$ & K & L \\
\hline H1 & ECG & BCG & ECG \\
H2 & BCG & BCG & BCG \\
H3 & BCG & ECG & BCG \\
H4 & BCG & BCG & BCG \\
H5 & ECG & BCG & BCG \\
H6 & BCG & ECG & BCG \\
H7 & BCG & ECG & BCG \\
H8 & BCG & BCG & BCG \\
H9 & BCG & BCG & BCG \\
H10 & BCG & BCG & BCG \\
H11 & BCG & ECG & BCG \\
P1 & ECG & BCG & BCG \\
P2 & BCG & BCG & BCG \\
A1 & BCG & BCG & BCG \\
A2 & BCG & BCG & BCG \\
A3 & ECG & BCG & ECG \\
A4 & BCG & BCG & BCG \\
\hline
\end{tabular}

Bold font highlights the best overall signal for each subject

$H$ healthy subject, $P$ patient, $A$ healthy subject with insulating layer between scalp and electrodes

The graph in Fig. 5 illustrates the percentage of incorrect markers resulting from the two detection methods (ECG or EEG-based) for each subject. In 2 cases (subject $\mathrm{H} 1$ and A3) the ECG peaks were perfectly identified and BCG detection was good as well (inaccurate detection $<10 \%$ ). With the exception of the two cases mentioned above, the detection based on the ECG signal gave a high number of wrong and/or missing markers, when compared to BCG.
These results are concordant with the findings reported in Table 1, i.e. the automatic algorithmic selection of the signal with the best detection. This means that the automatic selection can be trusted because it is always linked to the signal with the major number of corrected markers.

The GFP peak amplitude of the $E E G_{P A}$ was higher (mean $=73.8 \pm 28.4 \mu \mathrm{V}$ ) than GFP peak amplitude of the $E E G_{E C G}$ (mean $\left.=68.1 \pm 27.0 \mu \mathrm{V}, p=0.0012\right)$ and the width of $E E G_{P A}$ peak was smaller (mean $=77.3 \pm$ $15.1 \mathrm{~ms}$ ) than the width of $E E G_{E C G}$ peak (mean $=79.5 \pm$ $16.9 \mathrm{~ms}, p=0.0186$ ). These additional results suggested that even after correcting wrong and missing markers, BCG markers allow a better estimation of pulse artifacts than ECG peaks.

\section{Comparison of Electrode Sets for BCG Estimation}

Table 2 shows the comparison of the complete temporofacial set of electrodes with the reduced 64 channels subset of electrodes in all healthy subjects, patients and subjects with insulating layer between scalp and electrodes. The sensitivity and specificity of the reduced 64 channels subset is lowered compared to the complete set (95 and $97 \%$ instead of 97 and $98 \%$ ) but remains higher than the sensitivity and specificity of the detection using the ECG (76 and $49 \%$ ). The use of the complete temporo-facial set of electrodes is preferable when available because when some electrodes capture noise or epileptic activity, the mean signal of a larger number of electrodes decreases these confounding effects.

\section{Simulated Patient}

The topographic representation of epileptic spikes outside the magnetic environment showed clear independent anterior temporal (named RT) and posterior temporal (temporo-occipital, named RTO) activity.

The pulse artifact detection was performed on the BCG signal with good accuracy (93\% of correct detection). No epileptic spike was considered by the algorithm as PA peaks.

The addition of the artifacts related to the MR environment (both the GA and the PA), didn't alter the morphology or the voltage topography of epileptic events on the corrected EEG compared to the native recording as shown in Fig. 6a and b

\section{Discussion}

We developed an EEG-based method to characterize and detect the pulse artifact for an improved correction of EEG-fMRI data. The method is based on the topographic organization of the pulse artifact, consisting in an 


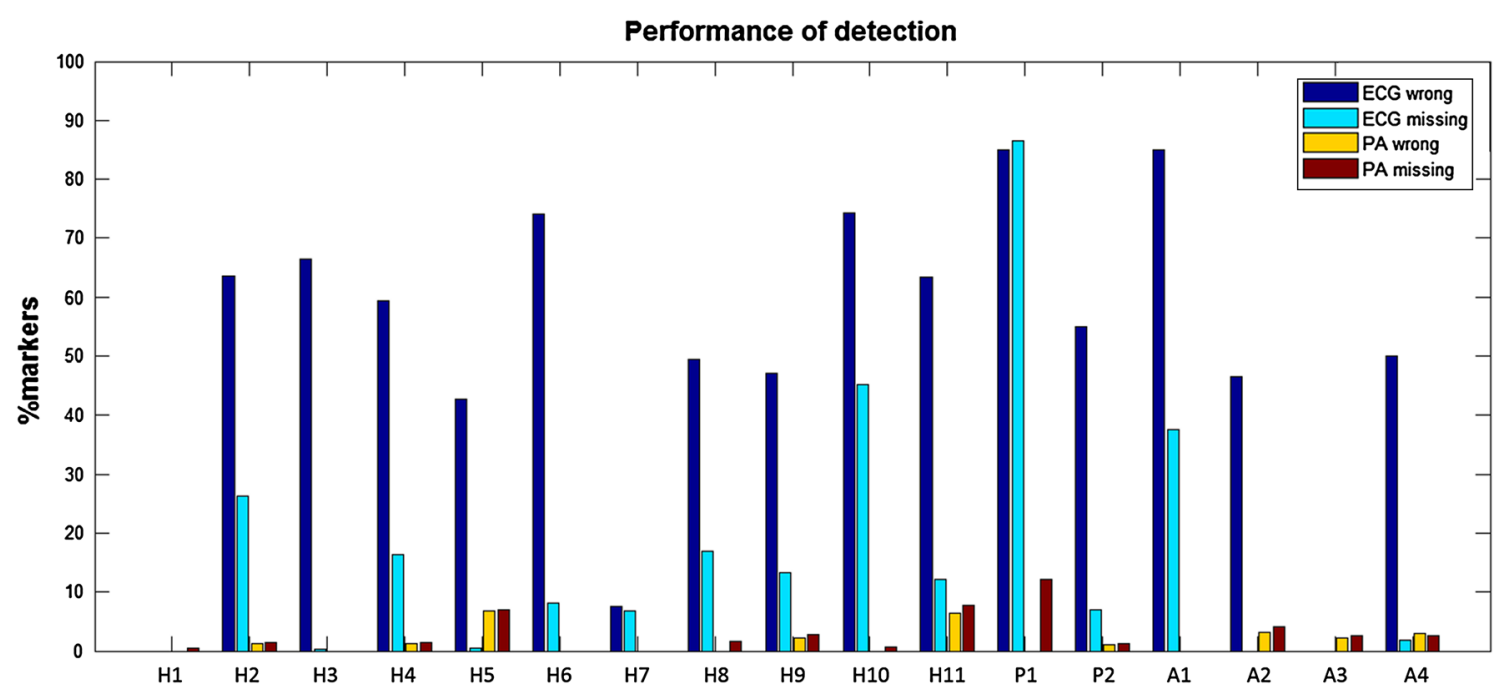

Fig. 5 For each subject, percentage of missing peaks and wrong peaks detection (calculated with respect to the total number of markers)

Table 2 Comparison of PA peaks detection accuracy using ECG, the complete temporo-facial set of electrodes and the reduced set of electrodes. In the lower table, mean and standard deviation values across subjects are reported

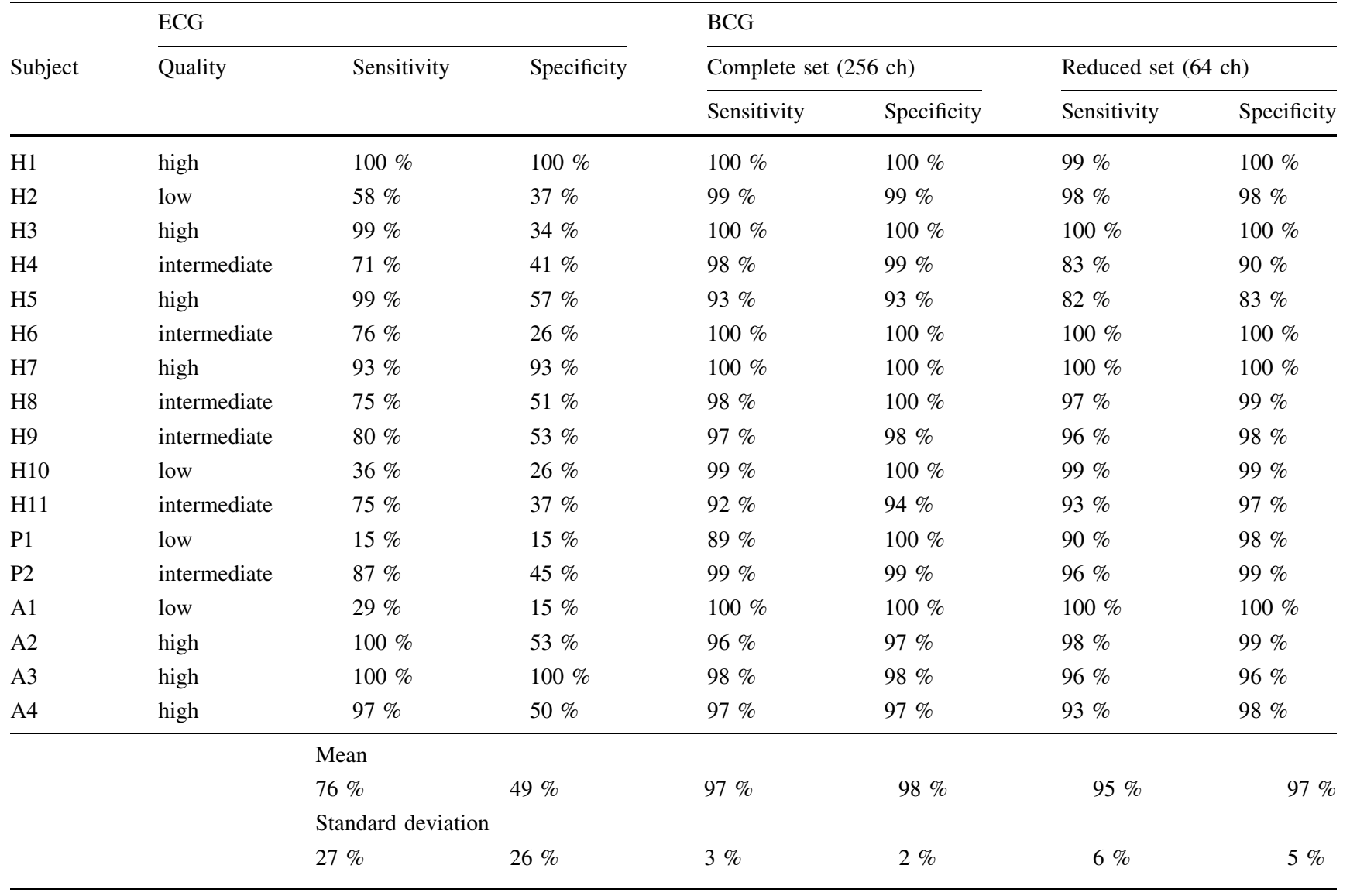

$H$ healthy subject, $P$ patient and $A$ healthy subject with insulating layer between scalp and electrodes

anti-symmetric scalp voltage potential topography. Therefore, this method directly detects the artifact on the scalp itself, i.e. in regions where it is predominant. Moreover, it doesn't require knowledge about the anatomy of scalp arteries, neither additional measuring equipment proposed in other studies such as the use of a Reference Layer 

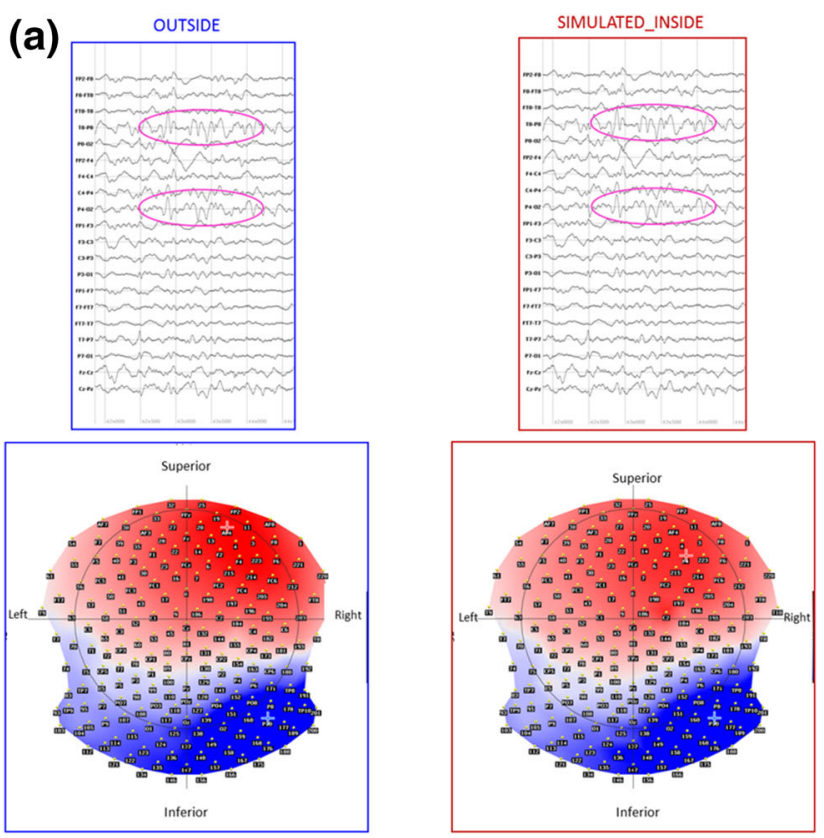

Fig. 6 Comparison of interictal epileptic topographies recorded outside MRI and during fMRI after artifacts correction. a Right temporo-occipital activity. Example of a series of polyspikes on the EEG surrounded by a pink line (top) and its corresponding averaged

(Chowdhury et al. 2014). Our technique can replace ECG, especially when it is of poor quality, due to poor electrode adherence or motion. This is of particular interest in children and cognitively impaired patients.

Using subjects with insulating layer between scalp and electrodes, we were able to replicate previous observations showing that the pulse artifact dynamics present polarity inversion between left and right electrodes (Debener et al. 2007; Mullinger et al. 2013).

Several reasons support our proposed EEG-based PA detection as a valuable alternative solution to ECG-based strategies.

- The GFP peak of the averaged signal aligned with PA markers, $E E G_{P A}$, is greater than the GFP peak of the averaged signal aligned with ECG markers, $E E G_{E C G}$. The latency of the PA with respect to the ECG peak varies across subjects and depends on their anatomical and physiological properties. Its intra-subject variability is also an important issue. Indeed, the more widespread technique used to remove PA from the EEG is AAS method which assumes a constant delay between the markers and PA. This assumption is not perfectly respected for ECG-based method but it applies to the BCG signal (where there is no latency). Moreover, even in cases where ECG-based detection is used, the BCG can provide a subject-specific estimation of this delay.

- The detection of PA peaks was more accurate using the BCG signal compared to the ECG signal, as shown by
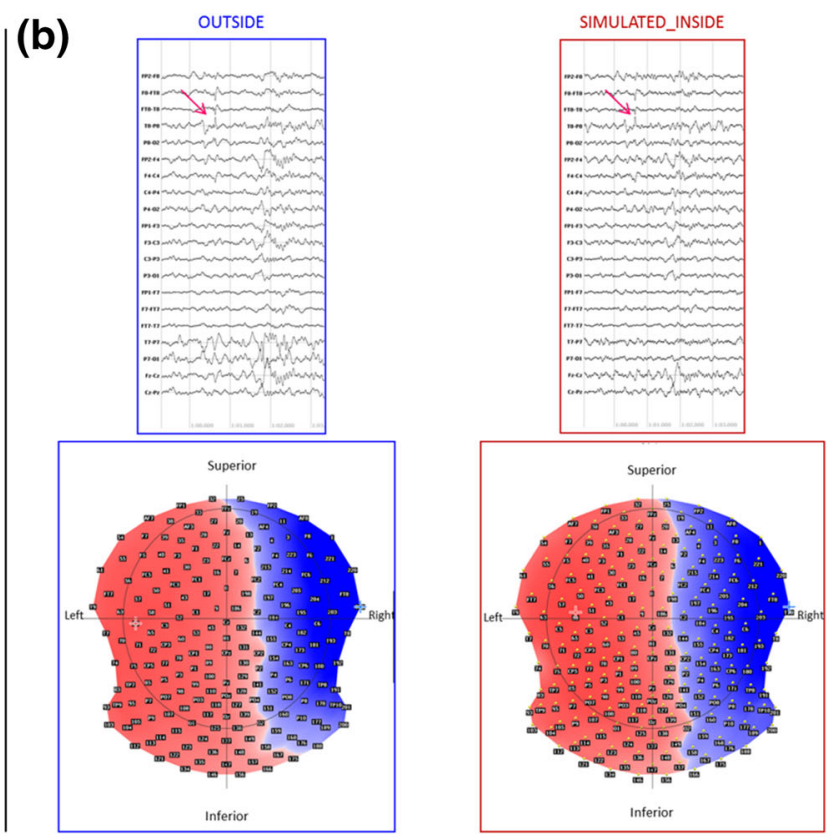

scalp topography (bottom). b Right anterior temporal activity. Example of a right temporal spikes on the EEG pointed by the red arrow (top) and its corresponding averaged scalp topography (bottom) (Color figure online)

the combined interpretation of the three criteria applied on automated peak detection (standard deviation, kurtosis and physiological confinement) and as confirmed by the visual inspection. In fact, the number of false positive $\left(\mathrm{F}_{\mathrm{P}}\right)$ and false negative $\left(\mathrm{F}_{\mathrm{N}}\right)$ events decreases with EEGbased detection. This makes the BCG model advantageous not only in terms of time but also because it reduces the subjective interpretation of the peaks.

- The higher amplitude and the sharper width of GFP peaks of $E E G_{P A}$ compared with the $E E G_{E C G}$ suggests that the PA peaks are more precisely identified on EEG with our method than with the help of ECG.

- In the majority of our cases, the waveform of the BCG was very well defined compared to that of the ECG, whose shape can be noisy after the gradient artifact correction (Fig. 7), which is a mandatory pre-processing step of the EEG acquired during fMRI.

- Detection of the PA directly on the head, i.e. in the center of the scanner bore, limits motion-induced currents. This is an advantage compared to the use of ECG chest electrodes located closer to the entrance of the bore, where they experience steeper changes in the static magnetic field as well as large breathing-related motions that amplify the artifacts. Breathing also induces motion at the level of the cheeks but with a less relevant contribution.

- The use of ECG during fMRI increases the risk of burn and heating (Dempsey and Condon 2001). At high field 
(a)

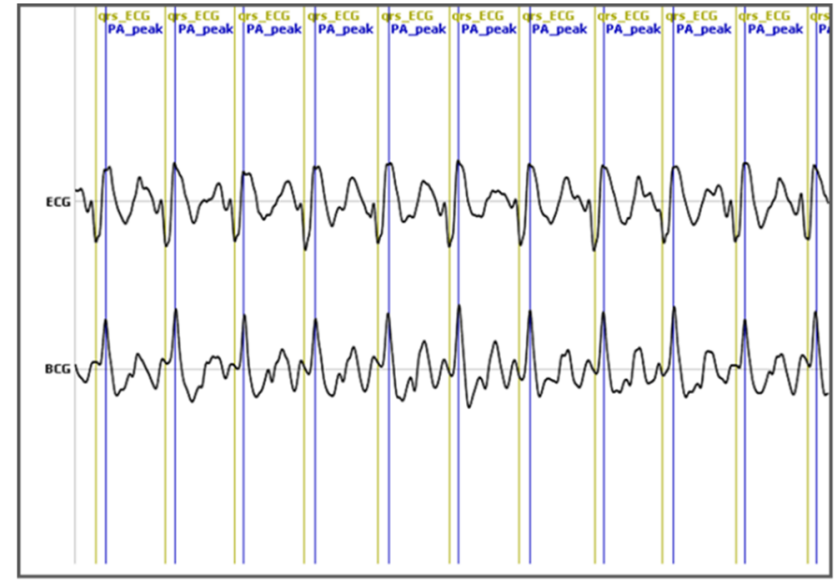

(b)

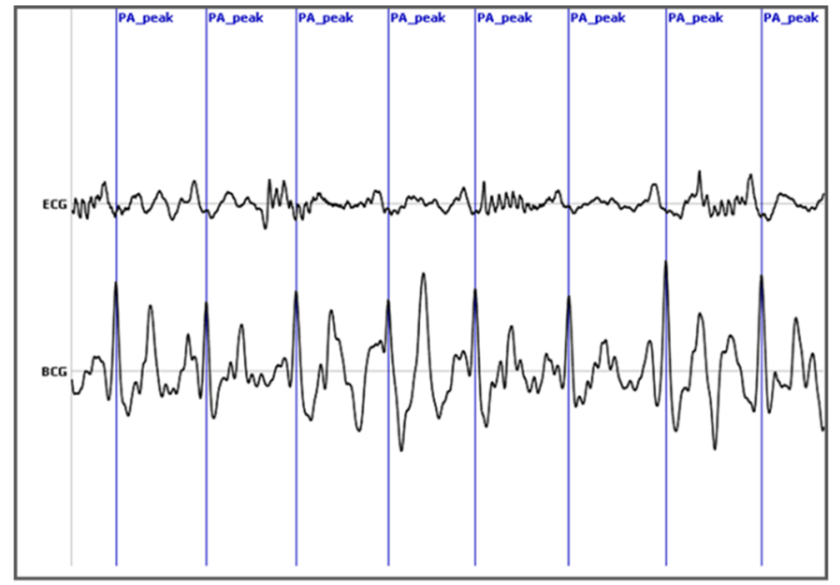

Fig. 7 Comparison between the ECG and the estimated BCG signal with markers indicating the occurrence of peaks in a recording with an ECG of good quality (a) and in a recording with an ECG of poor quality (b). In this last case, ECG-based method is not able to mark any peak

(e.g. $7 \mathrm{~T}$ ), the wavelength associated with radiofrequency pulse is shorter, and the risk of resonant antenna effect along ECG wires is greater (Dempsey et al. 2001). Consequently, the presented method is of great interest for future studies at $7 \mathrm{~T}$ and higher fields, because the estimated BCG signal amplitude will be stronger and easier to detect and because the recording of ECG will potentially become more dangerous.

- EEG-based correction remains the only way to have information about the cardiac related effect on the head in the absence of a reliable ECG recording, i.e. when ECG signal is of poor quality. This is a situation that is not rarely encountered in practice, caused by suboptimal ECG electrode contact, for example in men with hairy chest, placement of electrodes limited by breast anatomy or large irregular chest motion.

- The use of all temporo-facial electrodes demonstrated to be efficient in the definition of the BCG signal and in the ensuing peak selection, supporting the use of highdensity EEG system or, at least, additional facial electrodes. The quality of the signal derived from facial electrodes was improved by degreasing the skin fat and putting a fitting elastic bandage above the EEG cap, around the scalp and cheeks. With good impedance values, the influence of muscle artifact was reduced by averaging the complete set of 24 electrodes. Even if the use of a reduced set of electrodes alone is better in some cases (Table 2), the complete set of electrodes improve the quality of the mean signal by reducing the effect of artifact on a subset of electrodes.

- Importantly, our study showed that the EEG-based PA correction did not alter the identification of spikes and preserved their scalp topography compared to the spikes observed in the recording performed outside the magnetic environment. The acquisition and analysis of the EEG-based method should therefore be compatible with fMRI analysis guided by EEG topography (Grouiller et al. 2011), source activity (Vulliemoz et al. 2010) or simultaneous analysis of EEG-fMRI and Electric source Imaging (Vulliemoz et al. 2009). In clinical practice, current topographic EEG analyses with the 256-channel EEG-system used here are often computed by not considering the electrodes on the cheek (Brodbeck et al. 2009). The electrodes chosen for BCG estimation have only little overlap with those used for EEG analysis (6 electrodes in total). Therefore, these electrodes currently discarded for electric source imaging became fundamental for the correction of artifact.

\section{Limitations}

In case of frequent and/or ample head movements or other artifacts such as swallowing or facial muscular contraction that could be incidental or reflect an ictal event, the quality of the BCG signal could be deteriorated and not reliable to estimate PA artifact.

The application of the method described here is limited in datasets where less or no facial electrodes are used during EEG-fMRI recording. Nevertheless, we believe that in these cases, the theoretical principle to estimate the PA from the scalp remains reasonable. Adding few electrodes, like zygomatic electrodes, could provide the needed amount of signal to estimate the BCG and such strategy could be tested in a future study. The amplitude of the PA peak is proportional to the magnetic field strength. In our study, we demonstrated that it works well with $3 \mathrm{~T}$ scanner 
but the EEG-based method to remove the PA is probably less sensitive at $1.5 \mathrm{~T}$. However, we assume it enhances at higher magnetic field.

Acknowledgments This work was supported by Swiss National Science Foundation Grants 320030-141165 and 33CM30-140332 (SPUM Epilepsy) and by the Center for Biomedical Imaging (CIBM) of the Universities and Hospitals of Geneva and Lausanne, and the EPFL.

\section{References}

Allen PJ, Polizzi G, Krakow K, Fish DR, Lemieux L (1998) Identification of EEG events in the MR scanner: the problem of pulse artifact and a method for its subtraction. Neuroimage 8:229-239. doi:10.1006/nimg.1998.0361

Allen PJ, Josephs O, Turner R (2000) A method for removing imaging artifact from continuous EEG recorded during functional MRI. Neuroimage 12:230-239. doi:10.1006/nimg.2000. 0599

Benar C, Aghakhani Y, Wang Y, Izenberg A, Al-Asmi A, Dubeau F, Gotman J (2003) Quality of EEG in simultaneous EEG-fMRI for epilepsy. Clin Neurophysiol 114:569-580

Brandeis D, Naylor H, Halliday R, Callaway E, Yano L (1992) Scopolamine effects on visual information processing, attention, and event-related potential map latencies. Psychophysiology 29:315-336

Britz J, Van De Ville D, Michel CM (2010) BOLD correlates of EEG topography reveal rapid resting-state network dynamics. Neuroimage 52:1162-1170. doi:10.1016/j.neuroimage.2010.02.052

Brodbeck V, Lascano AM, Spinelli L, Seeck M, Michel CM (2009) Accuracy of EEG source imaging of epileptic spikes in patients with large brain lesions. Clin Neurophysiol 120:679-685. doi:10. 1016/j.clinph.2009.01.011

Brookes MJ, Mullinger KJ, Stevenson CM, Morris PG, Bowtell R (2008) Simultaneous EEG source localisation and artifact rejection during concurrent fMRI by means of spatial filtering. Neuroimage 40:1090-1104. doi:10.1016/j.neuroimage.2007.12.030

Brunet D, Murray MM, Michel CM (2011) Spatiotemporal analysis of multichannel EEG: CARTOOL. Comput Intell Neurosci 2011:813870. doi: $10.1155 / 2011 / 813870$

Chowdhury ME, Mullinger KJ, Glover P, Bowtell R (2014) Reference layer artefact subtraction (RLAS): a novel method of minimizing EEG artefacts during simultaneous fMRI. Neuroimage 84:307-319. doi:10.1016/j.neuroimage.2013.08.039

Christov II (2004) Real time electrocardiogram QRS detection using combined adaptive threshold. Biomed Eng Online 3:28. doi:10. $1186 / 1475-925 X-3-28$

Czisch M, Wehrle R, Kaufmann C, Wetter TC, Holsboer F, Pollmacher T, Auer DP (2004) Functional MRI during sleep: BOLD signal decreases and their electrophysiological correlates. Eur J Neurosci 20:566-574. doi:10.1111/j.1460-9568.2004. 03518.x

Debener S, Ullsperger M, Siegel M, Engel AK (2006) Single-trial EEG-fMRI reveals the dynamics of cognitive function. Trends Cogn Sci 10:558-563. doi:10.1016/j.tics.2006.09.010

Debener S, Strobel A, Sorger B, Peters J, Kranczioch C, Engel AK, Goebel R (2007) Improved quality of auditory event-related potentials recorded simultaneously with 3-T fMRI: removal of the ballistocardiogram artefact. Neuroimage 34:587-597. doi:10. 1016/j.neuroimage.2006.09.031

Debener S, Mullinger KJ, Niazy RK, Bowtell RW (2008) Properties of the ballistocardiogram artefact as revealed by EEG recordings at $1.5,3$ and $7 \mathrm{~T}$ static magnetic field strength. Int J Psychophysiol 67:189-199. doi:10.1016/j.ijpsycho.2007.05.015

Dempsey MF, Condon B (2001) Thermal injuries associated with MRI. Clin Radiol 56:457-465. doi:10.1053/crad.2000.0688

Dempsey MF, Condon B, Hadley DM (2001) Investigation of the factors responsible for burns during MRI. J Magn Reson Imaging 13:627-631

Felblinger J, Slotboom J, Kreis R, Jung B, Boesch C (1999) Restoration of electrophysiological signals distorted by inductive effects of magnetic field gradients during MR sequences. Magn Reson Med 41:715-721

Grouiller F, Vercueil L, Krainik A, Segebarth C, Kahane P, David O (2007) A comparative study of different artefact removal algorithms for EEG signals acquired during functional MRI. Neuroimage 38:124-137. doi:10.1016/j.neuroimage.2007.07.025

Grouiller F, Thornton RC, Groening K, Spinelli L, Duncan JS, Schaller K, Siniatchkin M, Lemieux L, Seeck M, Michel CM, Vulliemoz S (2011) With or without spikes: localization of focal epileptic activity by simultaneous electroencephalography and functional magnetic resonance imaging. Brain 134:2867-2886. doi:10.1093/brain/awr156

Koenig T, Melie-Garcia L (2010) A method to determine the presence of averaged event-related fields using randomization tests. Brain Topogr 23:233-242. doi:10.1007/s10548-010-0142-1

Lehmann D, Skrandies W (1980) Reference-free identification of components of checkerboard-evoked multichannel potential fields. Electroencephalogr Clin Neurophysiol 48:609-621

Lehmann D, Ozaki H, Pal I (1987) EEG alpha map series: brain micro-states by space-oriented adaptive segmentation. Electroencephalogr Clin Neurophysiol 67:271-288

Lemieux L, Allen PJ, Franconi F, Symms MR, Fish DR (1997) Recording of EEG during fMRI experiments: patient safety. Magn Reson Med 38:943-952

Mandelkow H, Halder P, Boesiger P, Brandeis D (2006) Synchronization facilitates removal of MRI artefacts from concurrent EEG recordings and increases usable bandwidth. Neuroimage 32:1120-1126. doi:10.1016/j.neuroimage.2006.04.231

Marques JP, Rebola J, Figueiredo P, Pinto A, Sales F, Castelo-Branco M (2009) ICA decomposition of EEG signal for fMRI processing in epilepsy. Hum Brain Mapp 30:2986-2996. doi:10.1002/ hbm. 20723

Mijovic B, Vanderperren K, Van Huffel S, De Vos M (2012) Improving spatiotemporal characterization of cognitive processes with data-driven EEG-fMRI analysis. Prilozi/Makedonska akademija na naukite i umetnostite, Oddelenie za bioloski i medicinski nauki $=$ Contributions/Macedonian Academy of Sciences and Arts, Section of Biological and Medical Sciences 33:373-390

Mullinger KJ, Havenhand J, Bowtell R (2013) Identifying the sources of the pulse artefact in EEG recordings made inside an MR scanner. Neuroimage 71:75-83. doi:10.1016/j.neuroimage.2012. 12.070

Neuner I, Arrubla J, Werner CJ, Hitz K, Boers F, Kawohl W, Shah NJ (2014) The default mode network and EEG regional spectral power: a simultaneous fmri-EEG study. PLoS One 9:e88214. doi:10.1371/journal.pone.0088214

Niazy RK, Beckmann CF, Iannetti GD, Brady JM, Smith SM (2005) Removal of FMRI environment artifacts from EEG data using optimal basis sets. Neuroimage 28:720-737. doi:10.1016/j. neuroimage.2005.06.067

Nierhaus T, Gundlach C, Goltz D, Thiel SD, Pleger B, Villringer A (2013) Internal ventilation system of MR scanners induces specific EEG artifact during simultaneous EEG-fMRI. Neuroimage 74:70-76. doi:10.1016/j.neuroimage.2013.02.016

Pittau F, Grouiller F, Spinelli L, Seeck M, Michel CM, Vulliemoz S (2014) The role of functional neuroimaging in pre-surgical 
epilepsy evaluation. Front Neurol 5:31. doi:10.3389/fneur.2014. 00031

Shin JH, Choi BH, Lim YG, Jeong DU, Park KS (2008) Automatic ballistocardiogram (BCG) beat detection using a template matching approach. Conference proceedings : Annual International Conference of the IEEE Engineering in Medicine and Biology Society IEEE Engineering in Medicine and Biology Society*** Conference 2008:1144-1146. doi:10.1109/IEMBS. 2008.4649363

Skrandies W (2007) The effect of stimulation frequency and retinal stimulus location on visual evoked potential topography. Brain Topogr 20:15-20. doi:10.1007/s10548-007-0026-1

Stern JM, Caporro M, Haneef Z, Yeh HJ, Buttinelli C, Lenartowicz A, Mumford JA, Parvizi J, Poldrack RA (2011) Functional imaging of sleep vertex sharp transients. Clin Neurophysiol 122:1382-1386. doi:10.1016/j.clinph.2010.12.049

Vanderperren K, De Vos M, Ramautar JR, Novitskiy N, Mennes M, Assecondi S, Vanrumste B, Stiers P, Van den Bergh BR, Wagemans J, Lagae L, Sunaert S, Van Huffel S (2010) Removal of BCG artifacts from EEG recordings inside the MR scanner: a comparison of methodological and validation-related aspects. Neuroimage 50:920-934. doi:10.1016/j.neuroimage.2010.01.010

Vulliemoz S, Thornton R, Rodionov R, Carmichael DW, Guye M, Lhatoo S, McEvoy AW, Spinelli L, Michel CM, Duncan JS,
Lemieux L (2009) The spatio-temporal mapping of epileptic networks: combination of EEG-fMRI and EEG source imaging. Neuroimage 46:834-843

Vulliemoz S, Lemieux L, Daunizeau J, Michel CM, Duncan JS (2010) The combination of EEG source imaging and EEG-correlated functional MRI to map epileptic networks. Epilepsia 51:491-505. doi:10.1111/j.1528-1167.2009.02342.x

Weikl A, Moshage W, Hentschel D, Schittenhelm R, Bachmann K (1989) ECG changes caused by the effect of static magnetic fields of nuclear magnetic resonance tomography using magnets with a field power of 0.5 to $4.0 \mathrm{~T}$. Z Kardiol 78:578-586

Yan WX, Mullinger KJ, Brookes MJ, Bowtell R (2009) Understanding gradient artefacts in simultaneous EEG/fMRI. Neuroimage 46:459-471

Yan WX, Mullinger KJ, Geirsdottir GB, Bowtell R (2010) Physical modeling of pulse artefact sources in simultaneous EEG/fMRI. Hum Brain Mapp 31:604-620. doi:10.1002/hbm.20891

Zotev V, Phillips R, Yuan H, Misaki M, Bodurka J (2014) Selfregulation of human brain activity using simultaneous real-time fMRI and EEG neurofeedback. Neuroimage 85(Pt 3):985-995. doi:10.1016/j.neuroimage.2013.04.126 